Heller, Monica. - Linguistic Minorities and Modernity. A sociolinguistic Ethnography. London-New York, Longman, 1998, $287 \mathrm{p}$.

\title{
Claudine Moïse
}

\section{(2) OpenEdition}

Journals

Édition électronique

URL : http://journals.openedition.org/etudesafricaines/130

DOI : $10.4000 /$ etudesafricaines. 130

ISSN : $1777-5353$

Éditeur

Éditions de l'EHESS

Édition imprimée

Date de publication : 1 janvier 2001

ISBN : 978-2-7132-1394-6

ISSN : 0008-0055

\section{Référence électronique}

Claudine Moïse, « Heller, Monica. - Linguistic Minorities and Modernity. A sociolinguistic Ethnography. London-New York, Longman, 1998, 287 p. », Cahiers d'études africaines [En ligne], 163-164 | 2001, mis en ligne le 22 novembre 2013, consulté le 05 février 2021. URL : http://journals.openedition.org/ etudesafricaines/130; DOI : https://doi.org/10.4000/etudesafricaines.130

Ce document a été généré automatiquement le 5 février 2021.

(c) Cahiers d'Études africaines 


\title{
Heller, Monica. - Linguistic Minorities and Modernity. A sociolinguistic Ethnography. London-New York, Longman, 1998, $287 \mathrm{p}$.
}

\author{
Claudine Moïse
}

1 Monica Heller a fait de son ouvrage une quête et nous livre dans un style fluide et vivant l'évolution actuelle d'une minorité linguistique, la minorité francophone de Toronto, confrontée aux nouvelles données mondiales; elle nous dit les changements sociaux, les rapports de force des langues en présence, l'anglais et le français dans ses variétés et ses idéologies. La minorité franco-ontarienne fut partie prenante du Canada français, catholique, replié sur la langue et la famille, dans sa certitude autarcique et linguistique. Dans les années 1970, avec le réveil nationaliste du Québec, la langue a été porteuse de l'idéologie unilingue centrée sur un État-nation, valeurs reproduites par les minorités linguistiques à travers les institutions unilingues obtenues de hautes luttes, comme l'école française. À ce propos, dans le premier chapitre, l'auteure montre, dans une belle synthèse théorique, le lien incontournable et paradoxal entre les minorités linguistiques et les États-nations: les minorités linguistiques construisent leur légitimité en reproduisant les valeurs du nationalisme qui les exclut: "Linguistic minorities are created by nationalisms which exclude them» (p. 7). Aujourd'hui, la donne a changé et dans un espace multiculturel comme Toronto, les locuteurs jouent, dans une perpétuelle tension et en contrepoids de l'idéologie linguistique portée, entre autres, par l'école, de nouvelles valeurs qui remettent finalement en question l'idéologie politique traditionnelle unilingue et nationale.

2 Monica Heller travaille depuis longtemps dans une perspective - trop rare encore en France - d'ethnolinguistique et d'ethnographie de la communication, sur les traces de la sociolinguistique américaine de Gumpez et de Hymes. C'est par une étude minutieuse $\mathrm{du}$ terrain qu'elle peut rendre compte de phénomènes plus vastes, du changement social, mais aussi de ses crispations : «Linguistic minorities thus illustrate particularly 
clearly the crisis of legitimacy that hypermodernity has brought us » (p. 15). Dans une province anglophone du Canada, l'Ontario, elle s'est rendue avec son équipe deux fois par semaine dans une école française de Toronto pendant presque quatre ans. Toronto est un bel exemple des nouvelles règles auxquelles se trouve confrontée la minorité francophone: communauté urbaine composite constituée d'une francophonie internationale, européenne ou africaine, et d'une francophonie canadienne, chacune avec des valeurs et des cultures différentes. L'enquête a été menée de 1991 à 1995 et représente un long travail d'ethnographie institutionnelle : observation dans les salles de classe, interviews avec le personnel enseignant et administratif, analyse des documents produits par l'école, participation aux activités.

Dans son ouvrage, l'auteure a su mettre en perspective l'étude de son corpus recueilli donc à l'école française Champlain de Toronto : elle montre avec brio (et c'est sans doute là l'aspect le plus original du livre) comment les tensions entre les langues en contexte scolaire sont emblématiques des idéologies linguistiques et des changements sociaux : "I chose to look at the problem of nationalism, identity and minorization through the lens of schooling. Schools are important sites of social and cultural reproduction» (p.18). L'école reste le lieu incontournable de reproduction d'une langue idéale, ligne de force des idéologies nationales, des États-nations, telles soutenues par le Québec et les minorités hors Québec dans les années 1970. Il y a donc la volonté affirmée par l'école d'afficher et d'imposer un français standard, image à laquelle doivent se rallier tous les élèves. Il s'agit alors de faire de l'unité dans la diversité. Cette vision $\mathrm{du}$ français repose sur une lutte identitaire en réaction contre l'assimilation au groupe anglais; mais elle ne prend pas en compte la tension qui existe entre les différents groupes francophones représentés à l'école, des francophones de la francophonie internationale, Africains ou Européens, des francophones du Canada, Québécois, Franco-Ontariens, enfin francophones à l'anglais dominant. Elle se manifeste dans la salle de classe par des corrections systématiques de la part des enseignants à l'égard des élèves ${ }^{1}$, reformulations des anglicismes ou tout simplement lutte contre l'emploi de l'anglais. Une façon de tendre vers un français épuré, homogène et fantasmé. Cette vision d'une langue française internationale, qui se veut prestigieuse et donne accès aux sphères économiques, rend compte des contradictions de l'école française franco-ontarienne: en effet, l'école doit sa légitimité, sa raison d'être première à la mobilisation franco-ontarienne, portée par une identité repérable et « authentique » et donc par le français vernaculaire de la province... français que l'école marginalise aujourd'hui. L'école rejette donc le français vernaculaire, marque pourtant d'une identité originelle, au profit d'un français standard au capital linguistique plus important. Cette contradiction prend forme dans la marginalisation que vivent les élèves franco-ontariens eux-mêmes.

4 Dans un style narratif particulièrement plaisant et par l'analyse du très riche corpus, Monica Heller montre aussi toutes les stratégies développées par les élèves pour s'accommoder, voire affronter, cette idéologie, reproduite en salle de classe et dans les murs de l'école, d'un monolinguisme imaginé. Face à ce mythe de l'homogène, luimême pris dans sa propre tension, se rejouent les conflits des langues dans leur réalité quotidienne, du français vernaculaire stigmatisé, à la valeur affirmée et revendiquée du bilinguisme dans un monde marqué par la mondialisation. Certains élèves et essentiellement des garçons, dans une belle réussite scolaire, ont fait avec fierté le choix du bilinguisme tout en reproduisant le discours de l'école basé sur un français international. Ils contrôlent l'espace social de l'école et s'insèrent avec succès dans 
celui de la société dominante tout en laissant dans l'insécurité linguistique les locuteurs d'un français vernaculaire ou même les franco-dominants tels les Québécois ou les jeunes femmes, notamment celles issues de l'immigration africaine. En ce sens, le français ne représente plus une identité commune forte mais devient la marque d'une nouvelle élite internationale.

Les interactions sont choisies avec pertinence et permettent alors de rendre compte de différents niveaux d'analyse. Du maniement micro-sociolinguistique des alternances d'abord. Monica Heller montre combien les alternances sont soit significatives, dans les reformulations qui les accompagnent, des normes linguistiques ${ }^{2}$, soit ont valeur de contre-norme de la part des élèves. Ensuite le corpus montre bien combien le jeu des langues, s'il est bien évidemment jeu de pouvoir au sein d'une situation institutionnelle, est également facteur de reproduction ou de transgression de l'ordre social, que d'une certaine façon il induit un maintien ou un renouvellement des frontières linguistiques et sociales.

6 Ainsi, à travers cette étude, Monica Heller élargit le champ de réflexion de la sociolinguistique. Elle questionne à partir d'une analyse précise de corpus le poids et l'évolution des idéologies linguistiques et donc politiques. Elle montre, en s'appuyant sur des méthodologies d'enquête et d'analyse solides, comment une sociolinguistique de terrain donne à comprendre d'une part les valeurs attribuées aux langues en présence (monolinguisme, recherche de la qualité, poids de la norme, etc.) et d'autre part les configurations sociales en mouvement et en changement. Mais sans doute, parce que Monica Heller nous donne une autre vision de la sociolinguistique, une sociolinguistique de terrain mâtinée de l'histoire pratique et théorique de l'ethnographie de la communication, et en perpétuelle réflexion sur l'évolution du monde qui nous entoure, nous aurions peut-être des leçons à en tirer en France pour nos propres travaux.

\section{NOTES}

1. Comme en témoigne cette interaction enseignante/élève (p.130) : enseignante: "Pourquoi lit-on? », élève : « Pour relaxer », enseignante : « Pour se détendre, relaxer c'est anglais. »

2. «Ça va être uh timé moi je pense c'est vraiment le le. chronométré je devrais dire / mot anglais / okay » (p. 125, extrait de corpus, interaction d'un enseignant). 